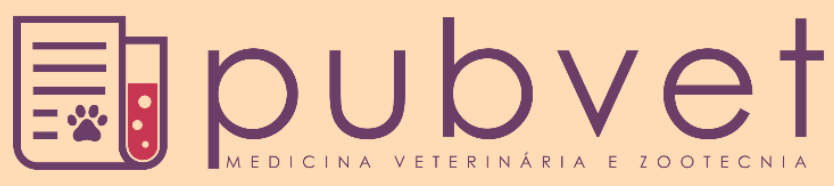

https://doi.org/10.31533/pubvet.v15n04a799.1-9

\title{
Estrongilídeos de ruminantes: Revisão
}

\author{
Francilara Costa do Nascimento ${ }^{1} \bullet$, Cristiane Lopes Mazzinghy ${ }^{2^{*}} \bullet$, Guilherme Augusto

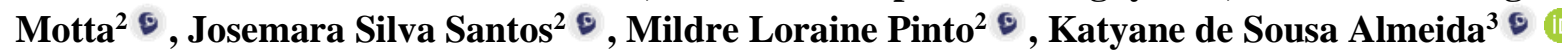 \\ ${ }^{I}$ Graduanda em Medicina Veterinária do Centro Universitário Luterano de Palmas. Palmas - TO Brasil. \\ 2 * Professores do Centro Universitário Luterano de Palmas, Departamento de Medicina Veterinária. Palmas - TO Brasil. \\ ${ }^{3}$ Professora da Universidade Federal do Tocantins, Departamento de Medicina Veterinária. Palmas - TO, Brasil. \\ *Autor para correspondência, cristiane.lopes@ceulp.edu.br
}

\begin{abstract}
Resumo. A pecuária brasileira está em constante desenvolvimento, contudo ainda enfrenta grandes problemas com parasitoses, diminuindo o desempenho do rebanho e consequentemente causando prejuízos econômicos. Os estrongilídeos são parasitos comumente observados no trato gastrointestinal destes animais e provocam danos como retardo do crescimento e diminuição de desempenho e isso se torna ainda mais evidente quando nos referimos aos pequenos ruminantes. Sabendo-se da importância destes parasitos gastrointestinais objetiva-se com esta revisão, discutir aspectos relacionados às parasitoses por estrongilídeos nos ruminantes bem como estratégias de controle.
\end{abstract}

Palavras-chave: Nematódeos, parasitoses, tricostrongílideos, verminoses

\section{Ruminant strongylides: Review}

Abstract. The Brazilian livestock is constantly developing; however, it still faces big issues with parasites, lowering cattle performance and consequently causing economic loss. Strongylides are parasites commonly observed in the gastrointestinal tract of these animals and cause damage such as growth retardation and decreased performance, and this becomes even more evident when we refer to small ruminants. Knowing the importance of these gastrointestinal parasites, this review aims to discuss aspects related to strongyloid parasites in ruminants as well as control strategies.

Keywords: Nematodes, parasitosis, tricostrongilídeos, verminosis

\section{Estrongílidos de rumiantes: Revisión}

Resumen. La ganadería brasileña está en constante desarrollo, pero aún enfrenta grandes desafíos problemas con los parásitos, disminuyendo el rendimiento del rebaño y, em consecuencia, causando pérdidas económicas. Los estrongílidos son parásitos que se observan comúnmente en el tracto gastrointestinal de estos animales y causan daños como retraso del crecimiento y disminución del rendimiento, y esto se hace aún más evidente cuando os referimos a pequeños rumiantes. Conociendo la importancia de estos parásitos gastrointestinales, esta revisión tiene como objetivo discutir aspectos relacionados con los parásitos estrongílideos em rumiantes, así como estrategias de control.

Palabras clave: Verminosis, parasitosis, tricostrongílidos, nematodos

\section{Introdução}

É difícil quantificar os impactos decorrentes das endoparasitoses no Brasil, pois existem poucos estudos experimentais que quantifiquem estas perdas (Costa et al., 2011). A maioria dos animais tem 
infecção subclínica, não apresentando sintomas e passando assim despercebidos pelos criadores. Os sinais clínicos podem aparecer mediante escassez de alimento, desmame, lotação de pastagem e infecções secundárias. Existem vários fatores que intensificam a predominância parasitária no rebanho como fatores climáticos, idade, raça, manejo, pastagem, tipo de criação e épocas em que foram coletados os dados (Gazda, 2006). Essas situações vão levar o animal a ter uma baixa na imunidade levando à manifestação de infecção clínica podendo-se observar nos mesmos, edema submandibular, abdominal e de extremidades, ascite, diarreia, abdômen distendido e mucosas pálidas (Chagas \& Veríssimo, 2008).

O grupo de nematódeos pertencentes a Subordem Strongylida provoca danos significativos aos ruminantes de acordo com a espécie de parasita, força da infecção, estado fisiológico e nutricional do hospedeiro. Na criação de pequenos ruminantes os danos ainda são mais severos, acarretando atraso no desenvolvimento do animal e até a morte dos mesmos pois são animais mais sensíveis a estas parasitoses (Vieira, 2008).

Dentro do grupo de helmintos gastrointestinais prevalentes na criação de bovinos, caprinos e ovinos pode-se observar os gêneros, Bunostomum spp; Cooperia spp; Haemonchus spp; Nematodirus spp; Oesophagostomum spp; Ostertagia spp; e Trichostrongylus spp. parasitando trato gastrointestinal destes ruminantes (Urquhart, 1996).

Existem alguns métodos clínicos e laboratoriais para análise de parasitos nos animais, mas são de pouca exatidão. $\mathrm{O}$ teste que apresenta melhores resultados apesar de também ter uma margem de instabilidade é o exame que determina quantidade de ovos por grama de fezes, obtido pelo teste de McMaster, que deve ser realizado antes e após o tratamento (Molento et al., 2004).

Sabendo-se da observação da intensidade parasitária em ruminantes nas propriedades do Brasil, o objetivo desta revisão é apresentar informações importantes sobre os parasitos e métodos de diagnóstico para estabelecer um bom manejo sanitário nas criações.

\section{Pecuária no Brasil}

A pecuária brasileira representa uma das principais atividades do agronegócio do país (ANUALPEC, 2020). A criação de gado para corte se destaca, com o país sendo o maior exportador no comércio mundial, ficando em segundo lugar no ranking de produção de carne bovina (FAPRI, 2020). Esta atividade pode ser caracterizada pelas diferentes maneiras de criação e comercialização dos rebanhos bovinos (Ferraz \& Felício, 2010; Prado, 2010).

$\mathrm{Na}$ atividade de pequenos ruminantes os aparatos tecnológicos são poucos no processo de produção, mas mesmo assim tem conseguido se manter em posição privilegiada no cenário do agronegócio (Maia et al., 1997; Silva Sobrinho, 2001). Isso está respaldado no aumento do consumo interno e em requerimento concreto de exportação de carne e pele para vários países, tal como na percepção de oportunidade de negócios que a atividade oferece (Aquino et al., 2016; Ribeiro, 1997; Sousa, 2007).

\section{Estrongilídeos de ruminantes}

O conceito de parasitismo é a interação entre duas espécies onde o parasita tem benefícios nessa associação, pois obtém nutrientes e abrigo no hospedeiro, causando-lhes prejuízos em alguma dimensão. Entre os parasitos observados nos animais pode-se citar os endoparasitas que mantem esta relação interespecífica habitando dentro do hospedeiro (Taylor et al., 2017).

As helmintoses gastrointestinais comumente ocorrentes nos ruminantes, caprinos e ovinos são provocadas por nematódeos que taxonomicamente encontram-se no Filo Nematoda, Ordem Strongylida, com algumas famílias e gêneros causando maiores ou menores danos aos hospedeiros (Monteiro, 2011).

Os estrongilídeos que acometem os ruminantes compõe gêneros distribuídos em várias famílias dentro da ordem Strongylida, sendo Haemonchus, Trichostrongylus e Ostertagia e Nematodirus pertencentes a família Trichostrongylidae, Oeshophagostomum à família Chabertiidae, e Bunostomum 
alocado dentro da família Ancylostomatidae (Furlong, 1999; Monteiro, 2011). A maioria desses parasitos são encontrados em regiões tropicais e subtropicais.

A espécie Haemonchus contortus é encontrada em grandes e pequenos ruminantes, são localizados no abomaso, e podem ser vistos em ocasião de necropsia pois medem de 2 a 3 centímetros. Eles possuem uma pequena cápsula bucal com a presença de uma fina lanceta, duas papilas cervicais, espículo curto e em forma de cunha. Nos machos, ainda se observa uma bolsa copuladora e as fêmeas tem apêndice vulvar. Ainda é possível a visualização dos ovários brancos enovelados em forma de caracóis em volta do intestino irrigado com sangue, levando animais a anemia severa que pode resultar em morte (Bowman, 2010; Monteiro, 2011).

O gênero Bunostomum é ocorrente em ruminantes. A espécie Bunostomum phlebotomum pode ser observada em ovinos e B. trigonocephalum em caprinos. São helmintos localizados no intestino delgado sendo que o verme pode chegar até $3 \mathrm{~cm}$ de comprimento. Possuem também uma grande cápsula bucal, e nas laterais um par de lâminas cortantes. Os ovos têm formato de elipse e possuem casca fina realizando eclosão momentos após a postura (Pinto, 1938).

Ostertagia spp. são helmintos encontrados em regiões de clima temperado e regiões subtropicais. A espécie Ostertagi ostertagi parasita abomaso de bovinos enquanto $O$. trifurcata e $O$. circumcincta são identificadas nos ovinos e caprinos. O gênero possui até um centímetro na fase adulta sendo observado no abomaso dos animais e envolvido em quadros de gastrite parasitária em ruminantes, com ocorrência de estágio larval nas glândulas gástricas. Os machos possuem gubernáculo e bolsa copuladora com presença de espículos curtos podendo ser bifurcado ou trifurcado. As fêmeas possuem vulva revertida com aumento cuticular, sendo que a larva L4 invade a mucosa do abomaso podendo ficar em hipobiose por algum período. As espécies se diferenciam pela quantidade de ramos do espículo observados no macho (Monteiro, 2011).

A espécie Trichostrongylus axei é encontrada nos bovinos, já os ovinos e caprinos são parasitados pelas espécies $T$. colubriformis, $T$. vitrinus, $T$ capricola, localizados em clima temperado e subtropicais. É um helminto localizado no abomaso dos animais, podendo atingir sete milímetros quando adultos. Os vermes possuem formato capiliforme, sulco excretor na região esofágica e espículos espessos e não ramificados. Na espécie T. axei tem comprimentos distintos e na fêmea a cauda é afilada, também não possuem apêndice vulvar colocando os ovos de forma longitudinal e em fileiras (Urquhart, 1996).

Os helmintos Cooperia oncophora, C. punctata, C. pectinata e C. surnabada parasitam intestino de bovinos, já as espécies $C$. surnabadae $C$. curticei são observadas nos ovinos e $C$. curticei nos caprinos. Tais espécies são localizados no intestino delgado dos ruminantes, esses vermes são semelhantes a corda de relógio, possuem pequenas vesículas cefálicas e estrias cuticulares em posição transversal no esôfago, não possuem gubernáculo, e os espículos tem tamanho parecidos com asa na região central e apresentam sulcos. Nas fêmeas são encontrados pequenos apêndices vulvares e uma cauda comprida e pontiaguda (Monteiro, 2011).

As espécies encontradas em bovinos são Nematodirus helvetianus e N.spathiger, já nos ovinos e caprinos observa-se $N$. battus, $N$. filicollis. $N$. spathiger. As espécies são comumente encontradas em regiões temperadas. Localizados no intestino delgado, os helmintos adultos são delgados e medem cerca de 2 centímetros de comprimento, e se entrelaçam parecendo algodão. Possuem pequenas vesículas cefálicas e sendo os machos portadores de longos espículos finos com extremidade fundidas. A fêmea apresenta cauda truncada com um pequeno espinho, sendo o ovo grande e incolor (Urquhart, 1996).

As espécies encontradas em bovinos são Oesophagostomum radiatum, e nos ovinos e caprinos são $O$. columbianum e $O$. venulosum. São espécies localizadas no ceco e cólon dos animais. Eles podem chegar até 2 centímetros de comprimento, e possuem cápsula bucal pequena geralmente circundadas com coroas lamelares, apresentam vesícula cefálica cuticular inflada terminando em um sulco cervical. As diferentes espécies são identificadas pelas posições das papilas cervicais e disposição da coroa lamelar. As larvas L3 depois de ingeridas pelo hospedeiro definitivo atravessam a mucosa do intestino grosso ou delgado, e ficam escondidas dentro de nódulos que podem ser vistos claramente, aguardando a transformação para L4 (Monteiro, 2011). 


\section{Métodos diagnósticos para estrongilídeos em ruminantes}

O modo de criação extensivo de animais, aumenta as chances de serem infectados por helmintos, principalmente os ruminantes jovens, mas nem sempre a infecção por esses helmintos representa danos severos. A força da infecção dos nematódeos gastrointestinais está associada com a idade, imunidade, espécie de helmintos, condições do ambiente e grau da infecção (Ueno, 1998).

Um dos principais métodos de diagnóstico dos estrongilídeos é a técnica coproparasitológica de Gordon \& Whitlock (1939). É um método quantitativo fundamentado na contagem de ovos por grama de fezes (O.P.G). Para realização do teste é necessária câmara especial designada câmara McMaster. Essa técnica possui como vantagem, a rapidez dos resultados, pois os ovos flutuam livres sem a presença de sujidades facilitando a contagem de ovos (Monteiro, 2011). A técnica é utilizada nos rebanhos para auxiliar o controle estratégico que irá reduzir a carga de parasitas dos animais, apresentando-se como ferramenta de monitoramento de carga parasitária no rebanho, possibilitando aplicação de boas práticas sanitárias, diminuindo a resistência anti-helmíntica e juntamente com esse exame deve ser feito também a coprocultura que identifica o gênero parasitário (Ferraz et al., 2019). Existem também outros métodos para averiguar o comprometimento parasitário no rebanho como o método FAMACHA (Molento et al., 2004). Utilizado para identificar a condição parasitária que o animal se encontra e os níveis de anemia provocada pela hemoncose. Ainda permite controlar a utilização de compostos antiparasitários a fim de promover a eficácia por longos períodos. Esse procedimento avalia a coloração da conjuntiva ocular do hospedeiro que será comparado a uma escala que varia do vermelho (grau 1) ao branco pálido (grau 5). Depois da observação os animais que tiveram coloração conjuntiva branco pálida deverão receber o tratamento (Amaducci et al., 2016).

O diagnóstico de infecções por estrongilídeos é baseado no histórico clínico, exibindo os sintomas e também a contagem ovos nas fezes. Em casos de altas cargas parasitárias de Haemonchus contortus, por exemplo, há comprometimento que pode levar a morte do animal. Assim, pode ser feita necropsia dando atenção ao abomaso e a medula dos ossos longos, mas na infecção hiperaguda somente o abomaso apresenta alterações. Na fase crônica da patologia o diagnóstico é mais complicado pois o animal estará desnutrido dificultando a confirmação (Urquhart, 1996). O diagnóstico da ostertagiose e cooperiose são feitos da mesma maneira, pela observação de sinais clínicos, e levando em consideração a estação do ano que pode ser favorável a infecção pela propagação das larvas. É necessário o teste McMaster e o teste dos níveis plasmáticos de pepsinogênio. Para observação do gênero Trichostrongylus é feita avaliação dos sinais clínicos apresentados, da necropsia em casos de óbitos e realização da técnica de McMaster. É importante fazer também cultura de fezes para detectar as características das larvas. O diagnóstico do Bunostomum é feito pela da contagem de ovos nas fezes, também pode ser feito durante o exame de necropsia (Pinto, 1938; Taylor et al., 2017; Urquhart, 1996).

O diagnóstico do Nematodirus e Oesophagostomum é feito pelo histórico de pastejo, pelos sinais clínicos e pela necropsia. Durante a infecção pelo gênero Oesophagostomum é possível visualizar nódulos purulentos das larvas encapsuladas nas mucosas do intestino. A contagem de ovos é pouco eficaz pois os sintomas aparecem no período pré-patente (Urquhart, 1996).

\section{Ciclo biológico}

Os helmintos passam por duas fases para completar o ciclo biológico. A primeira fase ocorre em vida livre e a segunda fase ocorre dentro do hospedeiro. Cada gênero de helminto tem particularidades no ciclo, por exemplo, nos Bunostomum a infecção se dá por outras vias sendo que há pela penetração

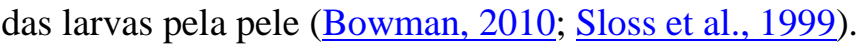

Mencionando o ciclo dos trichostrongilídeos, o grupo dentro do qual estão inseridos a maior parte dos gêneros causadores de infecção intestinais em ruminantes, as fêmeas põem centenas de ovos no tubo digestivo do hospedeiro, que expeli os ovos junto com as fezes na pastagem, e com auxílio da umidade, temperatura e ventilação, em um período de até 48 horas a larva de primeiro estágio (L1) se forma dentro do ovo. Após a eclosão do ovo, a L1 se alimenta de matéria orgânica, essa larva sofre então mais uma mudança se transformando em larva de segundo estágio (L2) que continua o processo de alimentação. A larva ainda sofre mais uma modificação se tornando uma larva de terceiro estágio 
ou L3 (Bowman, 2010; Sloss et al., 1999). As L3 são a forma infectante, muito resistentes ao ambiente pois são capazes de entrar em anidrobiose onde a atividade metabólica diminui e a capacidade de sobrevivência aumenta, com reservas que adquiriram durante o estádio de L1 e L2 (Amarante, 2015; Lettini \& Sukhdeo, 2006). Durante o tempo chuvoso espalham-se nas pastagens e assim infectam o hospedeiro. As larvas vão para o rúmen e lá perdem a bainha de proteção e se direcionam para o local de ação que pode ser o abomaso ou intestino delgado, e se tornarão adultas mudando de L4 para L5 (Monteiro, 2011).

\section{Sinais clínicos e patogenia}

A patogenia de ostertagiose ocorre quando há presença de muitos ovos, desencadeando infecções clínicas que causam as seguintes alterações: diminui a acidez do líquido abomasal e aumento da permeabilidade do epitélio do órgão às macromoléculas. Essas alterações causam o extravasamento do pepsinogênio para a circulação, acarretando seu aumento na concentração plasmática e perda da proteína plasmática para o lúmen do intestino provocando hipoalbuminemia. Isso vai provocar sinais clínicos como edema submandibular; diarreia aquosa profusa; perda de apetite e perda de peso (Taylor et al., 2017).

Devido os helmintos do gênero Haemonchus serem hematófagos e ingerir cada um cerca de $0,5 \mathrm{ml}$ de sangue durante o dia, ele provoca forte anemia hemorrágica nos pequenos ruminantes. Na hemoncose aguda ocorre uma diminuição do volume globular tornando a anemia evidente, e o quadro clínico pode piorar devido à perda de ferro e proteína no trato gastrintestinal, causando anorexia, levando a medula a total esgotamento, provocando a morte no animal. Nas ovelhas que estão em fase de amamentação pode ocorrer a morte dos cordeiros pois há uma diminuição abrupta na produção de leite. Durante a necropsia é possível observar pequenas lesões hemorrágicas na mucosa abomasal. Os sinais clínicos principais são anemia, edema submandibular, ascite, gastrite hemorrágica, queda de lã, fezes escuras e letargia (Urquhart, 1996).

A patogênese do Nematodirus se inicia com danos na mucosa intestinal, destacando a região do íleo. A transformação de L4 para L5 dura em média 12 dias, logo após isso a infecção causa erosão da mucosa e graves lesões nas vilosidades prejudicando a capacidade de troca dos líquidos e nutrientes intestinais, causando desidratação nos animais. Os sinais clínicos são diarreia com coloração verdeamarelada, tornando os animais sedentos, de forma que no pasto é possível observar os animais parados ao lado do bebedouro (Taylor et al., 2017). C. oncophora e C. curticei provocam retardo no desenvolvimento do animal. Este, depois de acometido vai desenvolver imunidade contra o nematódeo, e isso o torna resistente a uma segunda infecção. Já $C$. punctata e $C$. pectinata possuem ação mais severa contra os animais, pois rompem a superfície epitelial do intestino delgado ocasionando atrofia das vilosidades prejudicando a absorção. Os sinais clínicos manifestos decorrentes da infecção são: diarreia, edema submandibular, letargia e anorexia (Urquhart, 1996).

A patogênese do Oesaphagostomum se inicia quando a L3 migra para o interior da mucosa, desencadeando uma inflamação formando nódulos purulentos de 2 centímetros de diâmetro, com uma L4 no seu interior. Estes são visíveis no animal, em infecções graves levando ao quadro clínico de colite ulcerativa. Os sinais clínicos são diarreia com fezes verde escuras fétidas, perda de peso, prostração, emaciação e até a morte (Taylor et al., 2017).

As lesões provocadas pelo gênero Bunostomum ocorrem devido ao hábito hematófago do helminto. Nos animais jovens as larvas podem fazer penetração pela pele e isso leva o animal a desconforto e movimentação de patas com frequência, e também adquire o costume de lamber o local de penetração, onde há ocorrência de edema devido extravasamento de líquido no local. Isso vai ocasionar sinais clínicos como anemia, diarreia e retardo no desenvolvimento (Urquhart, 1996).

A patogenia do Trichostrongylus é caracterizada por lesões no abomaso, na parte do piloro e do fundo do estômago. Nota-se pequenas áreas irregulares, acúmulo de líquidos e lesões arredondadas de 1 a $2 \mathrm{~cm}$ proeminentes de coloração cinza claro. Pode-se observar úlceras rasas em infecções clínicas. Os sinais clínicos apresentados são perda de peso e diarreia (Taylor et al., 2017). 


\section{Epidemiologia}

A propagação das helmintoses está associada com o número de parasitos que estão presentes no ambiente, com o grau de infecção apresentado nos animais, o manejo realizado com este rebanho, fatores genéticos, clima e regiões. É essencial conhecer todos esses fatores para criar estratégias de controle que funcionem bem, evitando que os animais tenham resistência parasitária (Fortes, 2004).

A epidemiologia da ostertagiose nas regiões temperadas ocorrem da seguinte maneira; no inverno sobrevivem uma quantidade notável de larvas L3, e assim iniciarão a doença infectando os bezerros de uma forma que os animais tenham infecção subclínica, garantindo a contaminação dos pastos até o final do período de pastejo. Na primavera ocorre alta mortalidade das L3, tornando os pastos seguros. No verão as temperaturas aumentam e os ovos depositados na primavera se transformam em L3 podendo infectar os animais se ingeridas. No outono a temperatura cai e as L3 ingeridas não amadurecem inibindo o quarto estagio larval. Ainda assim os bezerros podem abrigar milhares de L4. Se grande parte das L4 se devolverem simultaneamente vão desencadear a doença na forma clínica (Urquhart, 1996).

A epidemiologia da Cooperia, no clima temperado se assemelha a Ostertagia, sofrendo hipobiose em fase L4, no término da primavera e verão. Nesse gênero as larvas não possuem grande potencial biótico e as L3 resistem melhor em clima árido. Os animais jovens costumam apresentar em algumas situações altas cargas parasitárias, já os adultos são considerados disseminadores por apresentarem poucos sinais clínicos e espalharem ovos nas pastagens (Taylor et al., 2017).

Sobre a epidemiologia dos Trichostrongylus, sabe-se que os ovos são embrionados e isso os tornam resistentes às variações climáticas, e as larvas também sofrem hipobiose auxiliando a propagação da doença no pasto no término do verão/outono, trazendo eventualmente problemas clínicos no rebanho durante o inverno e primavera (Urquhart, 1996).

Oesophagostomum tem epidemiologia recorrente em regiões tropicais e subtropicais. A L4 sobrevive por muitos dias nos nódulos da parede intestinal e a baixa imunidade torna difícil o controle, até o início da utilização de anti-helmínticos eficazes (Taylor et al., 2017).

Sobre Nematodirus, o principal aspecto sobre a epidemiologia desse gênero está no fato do ovo contendo a larva L3 conseguir sobreviver até dois anos em estágio de vida livre. Geralmente é no mês de maio e junho que o clima fica propício para a eclosão dos ovos, fazendo com que o evento ocorra simultaneamente causando infestação de L3 no pasto. E além disso os ovinos que têm poucos ovos nas fezes são capazes de desencadear infestações nas pastagens (Urquhart, 1996).

A disseminação do Bunostomum se inicia quando os ovos saem nas fezes do hospedeiro e se espalham no pasto, e com o auxílio da umidade do solo continuam evoluindo tornam-se larvas infectantes. Estas são protegidas pela cutícula, tornando-se resistentes na vegetação por muitos meses. Os ruminantes são infectados pelas larvas durante o pastejo e a ingestão de água. Assim, as larvas vão para o intestino onde continuam a modificação, e logo em seguida vão migrar para os pulmões. Os adultos são observados no intestino, iniciando a postura dentro do hospedeiro três a quatro semanas depois da ingestão da larva (Pinto, 1938).

A epidemiologia da hemoncose, causada pelo Haemonchus contortus ocorre muito nas regiões com temperatura alta, pois a umidade elevada auxilia o desenvolvimento das larvas. Para que a infecção se manifeste de maneira aguda é necessário primeiramente ter entre 2.000 e 20.000 ovos por gramas de fezes e a capacidade de sofrer hipobiose auxilia na sobrevivência das larvas, porém, só acontece em longos períodos de seca permitindo que a L4 sobreviva no hospedeiro em hipobiose, pois não se desenvolveriam no pasto árido (Urquhart, 1996).

\section{Controle}

O princípio de uma estratégia de controle por helmintos gastrointestinais, consiste em conhecer a epidemiologia destes bem como suas recorrências na região, e isso também auxiliará na elaboração de um programa de controle eficiente. Algumas técnicas utilizadas podem diminuir a carga parasitária no pasto, reduzindo os prejuízos causados por helmintoses no rebanho (Cezar et al., 2008). Uma das recomendações é fazer o manejo do rebanho e das pastagens corretamente, tendo conhecimento sobre 
condições epidemiológicas, entendendo como o clima vai atuar sobre os parasitos e o hospedeiro, e observando-se o manejo das pastagens. O pastejo rotacionado é um exemplo de manejo onde o pasto é divido em piquetes que vão receber animais, mas por pouco tempo. Isso será essencial para o combate aos parasitos, pois os animais dos piquetes vão ficar no local por tempo inferior ao desenvolvimento das larvas que estarão no ambiente. O intervalo da troca dos animais para um novo compartimento de alimentação vai tornar as larvas inviáveis promovendo descontaminação das pastagens (Silva, 2014).

Fazer a escolha de animais resistentes às parasitoses também é de suma importância para evitar danos às criações. $\mathrm{O}$ fornecimento de uma nutrição adequada contribui para a resistência do organismo animal às ações parasitárias (Cezar et al., 2008).

Uma das alternativas sustentáveis para o combate de parasitos é o controle biológico feito por fungos nematófagos, que são classificados como oportunistas, estes predadores parasitam os ovos e digerem as larvas aprisionando-as e consumindo seu conteúdo interno. Entre eles o que demonstra mais eficácia são os fungos predadores que conseguem diminuir as larvas do ambiente. Os gêneros de fungos nematófagos predadores mais eficazes são Artrhobotrys, Monacrosporium e Duddingtonia. De acordo com estudos esses fungos não prejudicam o meio ambiente (Cezar et al., 2008).

Os besouros coprófagos também fazem parte do controle biológico agindo na destruição das massas fecais nas pastagens evitando desenvolvimento de larvas nas fezes seguida de disseminação na pastagem (Mota et al., 2003). A espécie Digintonthophays gazela demonstra eficácia, se adaptando com facilidade em diferentes temperaturas e competindo com os nematódeos em sua fase de vida livre (Araújo et al., 2006). A utilização de plantas também pode ser empregada no controle de helmintos, um exemplo disso são espécies da família Fabacea, Espécies de forrageiras leguminosas desta família são ricas em metabólitos secundários como o tanino que pode exercer ação anti-helmíntica interferindo diretamente no ciclo natural dos helmintos (Hoste et al., 2006). Entretanto, tanto o tratamento fitoterápico, como o biológico ainda precisam de estudos que comprovem a eficácia na aplicação do combate aos nematódeos e que confiram segurança à saúde dos animais, com ausência de toxicidade (Cezar et al., 2008).

\section{Considerações finais}

Os problemas sanitários no rebanho decorrente das parasitoses gastrintestinais são acompanhados de perdas produtivas importantes ao produtor. Estes impactos são evidenciados principalmente na ovino e caprinocultura e muitas vezes não é documentado na bovinocultura. Assim, o conhecimento de métodos diagnósticos e de estratégias de controle permitem que novas alternativas surjam como proposta eficaz para melhorar a produtividade, melhorando o desempenho dos animais da criação e evitando quadros de resistência parasitária nas propriedades.

\section{Referências}

Amaducci, A. G., Borges, J. L., Sitko, M. D., Martines, T. T., Silva, J. C. A., Santos, A. P. Z., Ferreira, G. A., de Almada, A. F. B. \& Junior, R. P. (2016). Parâmetros sanguíneos e OPG (ovos por grama de fezes) de ovelhas mestiças da raça Dorper em diferentes graus do método Famacha. Arquivos de Ciências Veterinárias e Zoologia da UNIPAR, 19(4), 221-225. DOI: https://doi.org/10.25110/arqvet.v19i4.2016.6100.

Amarante, A. F. T. (2015). Os parasitos de ovinos. Editora da UNESP.

ANUALPEC. (2020). Anuário da Pecuária Brasileira (20th ed., Vol. 1). Instituto FNP.

Aquino, R. S., Lemos, C. G., Alencar, C. A., Silva, E. G., Lima, R., Gomes, J. A. F. \& Silva, A. F. (2016). A realidade da caprinocultura e ovinocultura no semiárido brasileiro: um retrato do sertão do Araripe, Pernambuco. PUBVET, 10(4), 271-281. DOI: https://doi.org/10.22256/pubvet.v10n4.271-281

Araújo, J. V, Assis, R. C. L., Campos, A. K. \& Mota, M. A. (2006). Efeito antagônico de fungos predadores dos gêneros Monacrosporium, Arthrobotrys e Duddingtonia sobre larvas infectantes de Cooperia sp. e Oesophagostomum sp. Arquivo Brasileiro de Medicina Veterinária e Zootecnia, 58(3), 373-380. DOI: https://doi.org/10.1590/s0102-09352006000300014 
Bowman, D. D. (2010). Parasitologia veterinária. Elsevier.

Cezar, A. S., Catto, J. B. \& Bianchin, I. (2008). Controle alternativo de nematódeos gastrintestinais dos ruminantes: atualidade e perspectivas. Ciência Rural, 38(7), 2083-2091. DOI: https://doi.org/10.1590/s0103-84782008000700048

Chagas, A. C. S. \& Veríssimo, C. J. (2008). Principais enfermidades e manejo sanitário de ovinos. Embrapa Pecuária Sudeste, 1(1), 70.

Costa, V. M. M., Simões, S. V. D. \& Riet-Correa, F. (2011). Controle das parasitoses gastrintestinais em ovinos e caprinos na região semiárida do Nordeste do Brasil. Pesquisa Veterinária Brasileira, 31(1), 65-71. DOI: https://doi.org/10.1590/s0100-736x2011000100010

FAPRI. (2020). Food and Agricultural Policy Research Institute (W. A. O. Database (ed.)). Food and Agricultural Policy Research Institute; Iowa State University and University of MissouriColumbia. http://www.fapri.iastate.edu/tools/outlook.aspx

Ferraz, A., Castro, T. A., Evaristo, T. A., Recuero, A. L. C., Dallmann, P. R. J., Motta, J. F. \& Nizoli, L. Q. (2019). Levantamento de parasitos gastrintestinais diagnosticados em ovinos pelo Laboratório de Doenças Parasitárias da Universidade Federal de Pelotas (Brasil), nos anos de 2015 a 2017. Revista Brasileira de Zoociências, 20(1), 1-7. DOI: https://doi.org/10.34019/2596$\underline{3325.2019 . v 20.24786}$

Ferraz, J. B. S. \& Felício, P. E. (2010). Production systems - An example from Brazil. Meat Science, 84(2), 238-243. https://doi.org/10.1016/j.meatsci.2009.06.006.

Fortes, E. (2004). Parasitologia veterinária (4th ed.). Editora ícone.

Furlong, J. (1999). Diagnosis of the susceptibility of the cattle tick, Boophilus microplus, to acaricides in Minas Gerais state, Brazil. 4. Seminario Internacional de Parasitología Animal, 1, 41-46.

Gazda, T. L., Piazzetta, R. G., Dittrich, J. R., Monteiro, A. L. G. \& Soccol, V. T. (2012). Distribuição de larvas de nematódeos gastrintestinais de ovinos em pastagens de inverno. Ciência Animal Brasileira, 13(1), 85-92. https://doi.org/10.5216/cab.v13i1.4025

Gordon, H. M. \& Whitlock, H. V. (1939). A new technique for counting nematode eggs in sheep faeces. Journal of the Council for Scientific and Industrial Research, 12(1), 50-52.

Hoste, H., Jackson, F., Athanasiadou, S., Thamsborg, S. M. \& Hoskin, S. O. (2006). The effects of tannin-rich plants on parasitic nematodes in ruminants. Trends in Parasitology, 22(6), 253-261. DOI: https://doi.org/10.1016/j.pt.2006.04.004

Lettini, S. E. \& Sukhdeo, M. V. K. (2006). Anhydrobiosis increases survival of trichostrongyle nematodes. Journal of Parasitology, 92(5), 1002-1009. DOI: https://doi.org/10.1645/ge-784r.1

Maia, M. S., Maciel, F. C. \& Lima, G. F. C. (1997). Criação de caprinos e ovinos: recomendações básicas de manejo. Natal: SEBRAE/RN, EMPARN.

Molento, M. B., Tasca, C., Gallo, A., Ferreira, M., Bononi, R. \& Stecca, E. (2004). Método Famacha como parâmetro clínico individual de infecção por Haemonchus contortus em pequenos ruminantes. Ciência Rural, 34(4), 1139-1145. DOI: https://doi.org/10.1590/s0103$\underline{84782004000400027}$

Monteiro, S. G. (2011). Parasitologia na medicina veterinária (Vol. 1). Roca.

Mota, M. A., Campos, A. K. \& Araújo, J. V. (2003). Controle biológico de helmintos parasitos de animais: estágio atual e perspectivas futuras. Pesquisa Veterinária Brasileira, 23(3), 93-100. DOI: https://doi.org/10.1590/s0100-736x2003000300001

Pinto, C. (1938). Sobre os gêneros" Bunostomum" Railliet, 1902 e" Bustomum" Lane, 1917.(Nematoda-Ancylostomidae). Revista Da Faculdade de Medicina Veterinária, Universidade de São Paulo, 1(1), 3-17. DOI: https://doi.org/10.11606/issn.2318-5066.v1i1p3-17

Prado, I. N. (2010). Produção de bovinos de corte e qualidade da carne (Vol. 1, Issue 1). Eduem.

Ribeiro, S. D. A. (1997). Caprinocultura: Criação Racional de Caprinos. Nobel.

Silva, H. M. (2014). Nematodioses gastrointestinais de caprinos: uma revisão. Revista de Ciências Agroveterinárias, 13(2), 199-208.

Silva Sobrinho, A. G. (2001). Criação de ovinos. Funep. 
Sloss, M. W., Zajac, A. M. \& Kemp, R. L. (1999). Parasitologia clínica veterinária (2a Ed.). Guanabara Koogan.

Sousa, W. H. (2007). O agronegócio da caprinocultura de corte no Brasil. Tecnologia \& Ciências Agropecuária, 1(1), 51-58.

Taylor, M. A., Coop, R. L. \& Wall, R. L. (2017). Parasitologia Veterinária. Guanabara Koogan.

Ueno, H. (1998). Manual para diagnóstico das helmintoses de ruminantes. UFRGS, JICA.

Urquhart, G. M. (1996). Parasitologia veterinária (2nd ed.). Guanabara Koogan.

Vieira, L. S. (2008). Métodos alternativos de controle de nematóides gastrintestinais em caprinos e ovinos. Tecnologia \& Ciência Agropecuária, 3, 49-56.

\section{Histórico do artigo:}

Recebido: 15 de outubro de 2020. Aprovado: 9 de dezembro de 2020

Disponível online: 15 de março de 2021
Licenciamento: Este artigo é publicado na modalidade Acesso Aberto sob a licença Creative Commons Atribuição 4.0 (CC-BY 4.0), a qual permite uso irrestrito, distribuição, reprodução em qualquer meio, desde que o autor e a fonte sejam devidamente creditados. 